\title{
A Polymer with Mechanochemically Active Hidden Length
}

\author{
Yancong Tian," Xiaodong Cao," Xun Li," Huan Zhang, Cai-Li Sun, Yuanze Xu, Wengui Weng,* \\ Wenke Zhang,* and Roman Boulatov*
}

Cite This: J. Am. Chem. Soc. 2020, 142, 18687-18697

Read Online

ACCESS | LWl Metrics \& More | 回 Article Recommendations

ABSTRACT: Incorporating hidden length into polymer chains can improve their mechanical properties, because release of the hidden length under mechanical loads enables localized strain relief without chain fracture. To date, the design of hidden length has focused primarily on the choice of the sacrificial bonds holding the hidden length together. Here we demonstrate the advantages of adding mechanochemical reactivity to hidden length itself, using a new mechanophore that integrates $(Z)$-2,3-diphenylcyclobutene1,4-dicarboxylate, with hitherto unknown mechanochemistry, into macrocyclic cinnamate dimers. Stretching a polymer of this mechanophore more than doubles the chain contour length

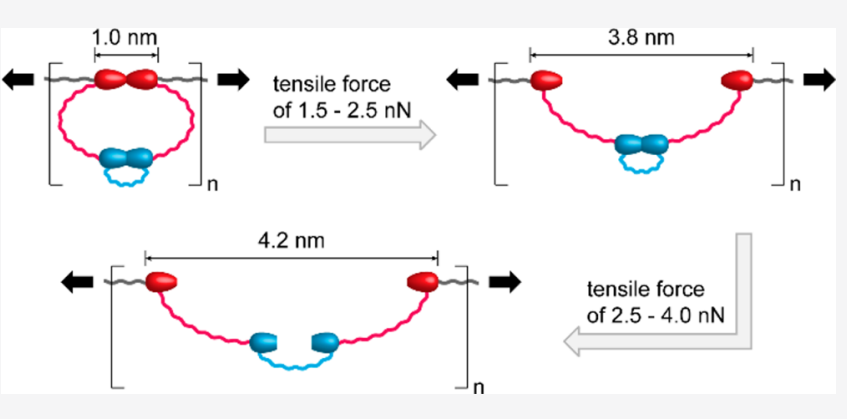
without fracture. DFT calculations indicate that the sequential dissociation of the dimer, followed by cyclobutene isomerization at higher forces yields a chain fracture energy 11 times that of a simple polyester of the same initial contour length and preserves high energy-dissipating capacity up to $\sim 3 \mathrm{nN}$. In sonicated solutions cyclobutene isomerizes to two distinct products by competing reaction paths, validating the computed mechanochemical mechanism and suggesting an experimental approach to quantifying the distribution of single-chain forces under diverse loading scenarios.

\section{INTRODUCTION}

Polymer hidden length is a portion of a polymer backbone that does not contribute to the end to end distance because it is contained within a macrocyclic loop held together by a chemical bond between two nonadjacent backbone atoms. When a polymer chain containing hidden length is stretched, the backbone within each loop remains strain-free until and unless the bond that holds the loop together ("sacrificial bond") dissociates. ${ }^{1-4}$ This dissociation abruptly increases the contour length of the stretched chain and redistributes the load away from overstretched segments. Single-molecule force experiments provide clear evidence of such load redistribution in a stretched chain upon release of hidden length. ${ }^{5-8}$

Backbone loops held by ionic or $\mathrm{H}$ bonds are common in biological structural materials and are well recognized to contribute to the remarkable mechanical properties of the bone and shells, muscle, and biological adhesives. ${ }^{4,9}$ These weak sacrificial bonds dissociate in response to modest loads (singlechain force $<0.4 \mathrm{nN}$ ) and re-form at practical time scales once the load dissipates. ${ }^{10,11}$ The rarer examples of covalent sacrificial bonds $3,6,8,12-14$ manifest irreversible chain elongation but only at much larger loads (single-chain force $>1 \mathrm{nN}$ ). They are designed to compete with mechanochemical chain fracture and to occur only in overstressed regions of loaded materials, ${ }^{15}$ which are at the highest risk of macroscopic failure. The kinetics of sacrificial bond dissociation is a key determinant of the mechanical behavior of polymers with hidden lengths. ${ }^{1}$ The known hidden-length polymers have a single mechano- chemical element: the sacrificial bond(s). A possibility that combining sacrificial bonds with additional mechanoresponsive moieties could improve the stress-responsive properties of polymers at single-chain or bulk levels has not been discussed in the literature.

Here we describe computational and experimental evidence that incorporating mechanochemical reactivity into hidden length both increases chain fracture energy and extensibility and provides a simple means of integrating additional productive mechanochemical responses into the polymer. Although a few mechanophores containing multiple fused rings are known ${ }^{12,16}$ in which multiple sacrificial bonds dissociate sequentially, their molecular architecture and the relative mechanochemical reactivity of their sacrificial bonds make them unsuitable for systematic studies of mechanochemically active hidden lengths. We therefore designed and characterized the tricyclic monomer 1 (Figure 1), which exploits the uniquely flexible molecular architecture of cinnamate dimer (red) macrocycles to incorporate a new mechanophore, diphenylcyclobutene (blue), into the hidden length. At $\sim 2 \mathrm{nN}$ the dimer dissociates on a $100 \mathrm{~ms}$ time scale,

Received: August 27, 2020

Published: October 16, 2020 


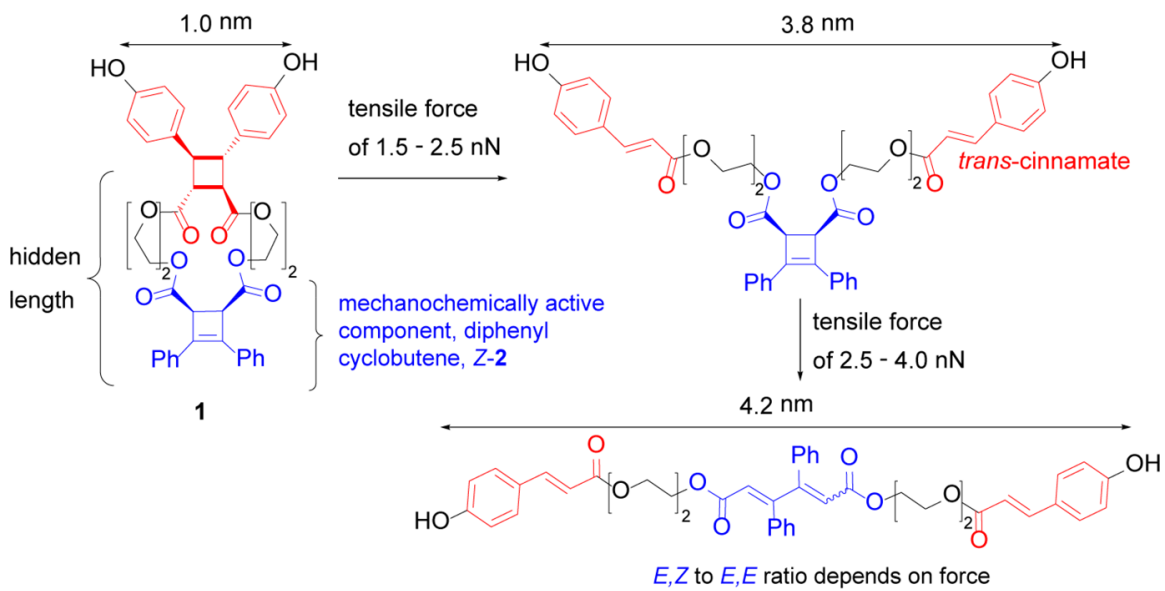

Figure 1. Chemical realization of mechanochemically active hidden length. If the mechanochemically active moiety in the hidden length (blue) is more resistant to applied force than the sacrificial bonds holding the hidden length (bold bonds of the red moiety in $\mathbf{1}$ ), the mechanophore undergoes stepwise elongation, retaining the energy-dissipating capacity to high force.
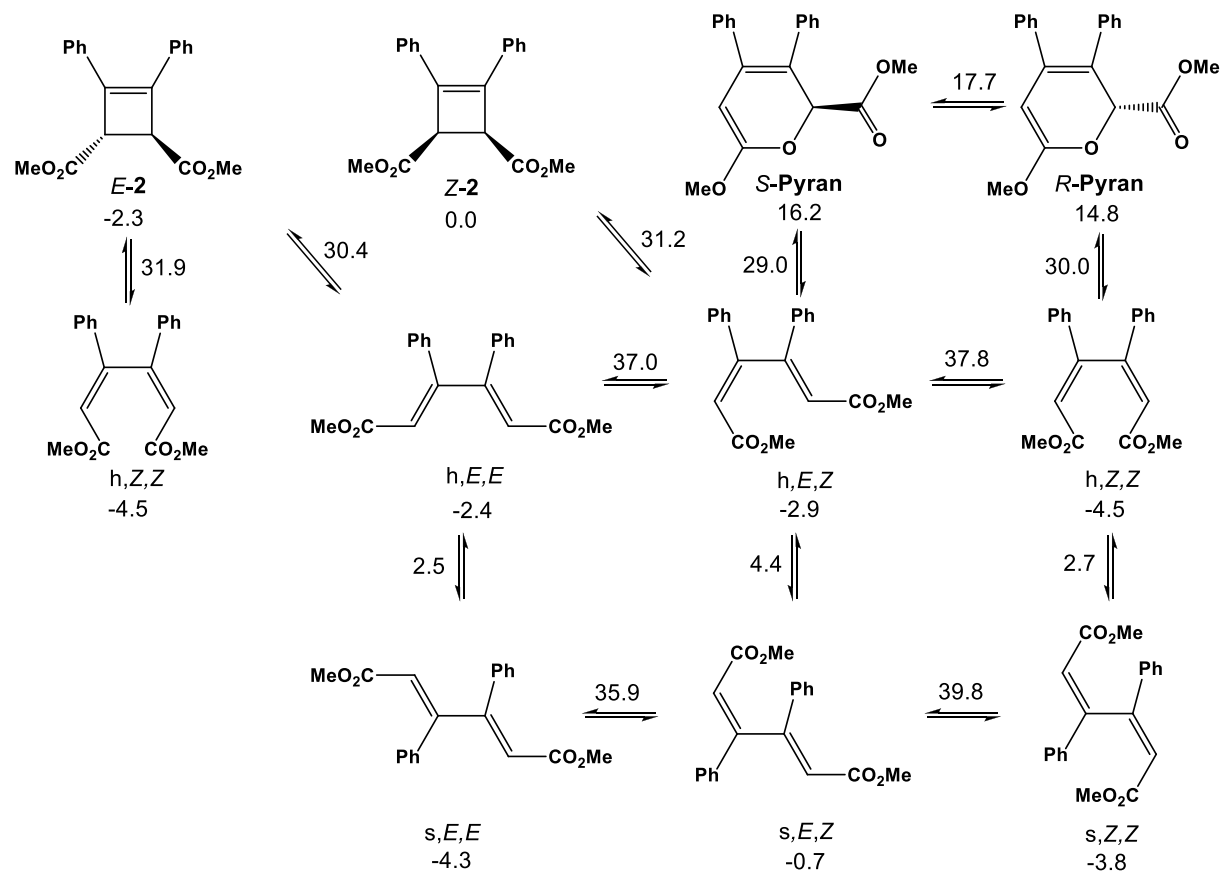

Figure 2. Calculated strain-free mechanism and energetics of isomerization among cyclobutene 2 and the corresponding dienes. The values given are standard free energies of the energy minima (beneath structures) or transition states (over the arrows) relative to that of Z-2 at the CAMB3LYP/6-311+G(d)/CPCM=DMSO level.

elongating the mechanophore by $2.8 \mathrm{~nm}$ and increasing the fracture energy of its polymer $>7$-fold relative to an aliphatic polyester of the same initial contour length. Unlike all previously reported polymers with hidden lengths, ${ }^{6,12,17}$ a polymer of 1 retains its energy-dissipating capacity even at $>2.5$ $\mathrm{nN}$ by virtue of containing another mechanophore that is stable at this force. Stretching beyond $2.5 \mathrm{nN}$ isomerizes cyclobutene (blue), elongating the polymer and increasing its fracture energy by an additional $0.4 \mathrm{~nm}$ per monomer and $40 \%$, respectively. Cinnamate dimers enable the facile synthesis of the required molecular architecture and make the remodeled material optically healable. ${ }^{6}$ The unprecedented mechanochemical stability of diphenylcyclobutene enables energy dissipation up to high force, and the competing isomerization mechanisms potentially allow much more accurate estimates of single-chain forces in loaded bulk samples than is achievable with any other strategy proposed to date. ${ }^{18}$

In the rest of the paper we first present a detailed analysis of the computed kinetics and mechanisms of isomerization of cyclobutene 2, which explains our choice of this mechanophore and contributes to the ongoing discussion about the role of orbital-symmetry (Woodward-Hoffmann) rules in governing mechanochemical electrocyclic reactions, ${ }^{19-21}$ followed by a discussion of the calculated chain micromechanics of the polymer of 1, P1, that illustrates the advantage of making the hidden length mechanochemically active. Finally, we demonstrate that $\mathbf{P 1}$ is readily accessible synthetically and its experimental mechanochemistry agrees with the quantumchemical predictions. 
A
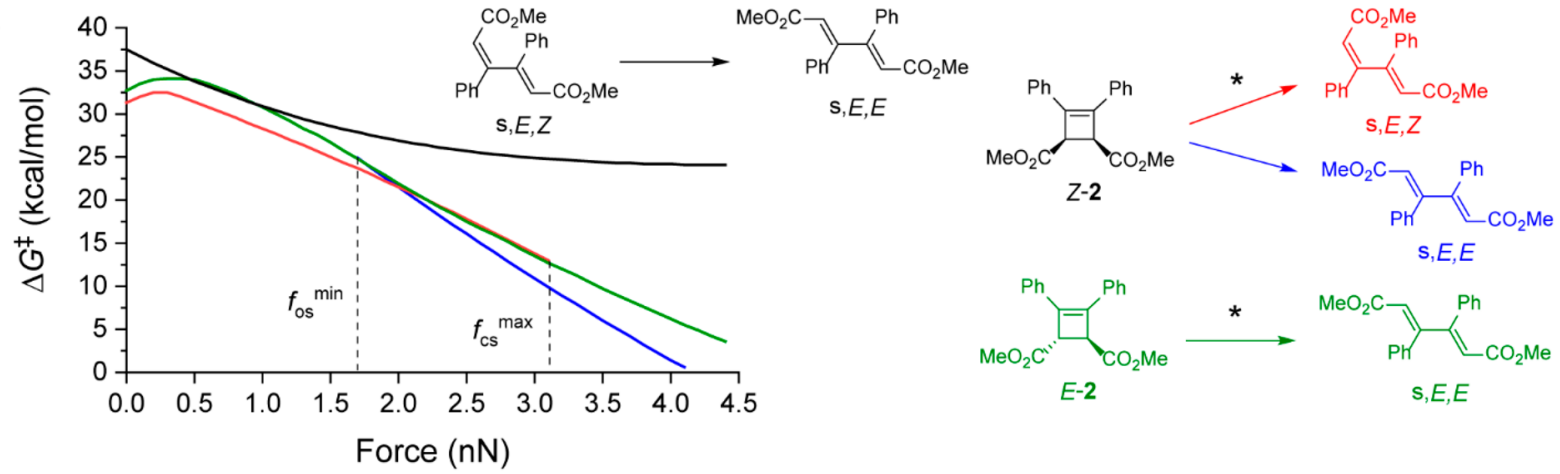

B

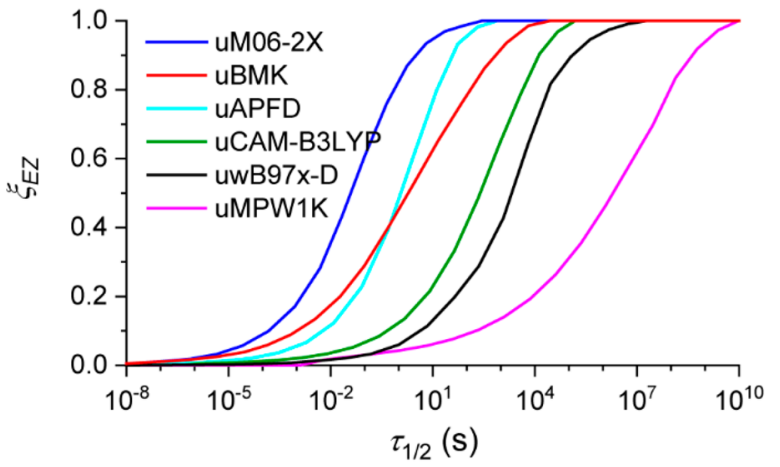

D

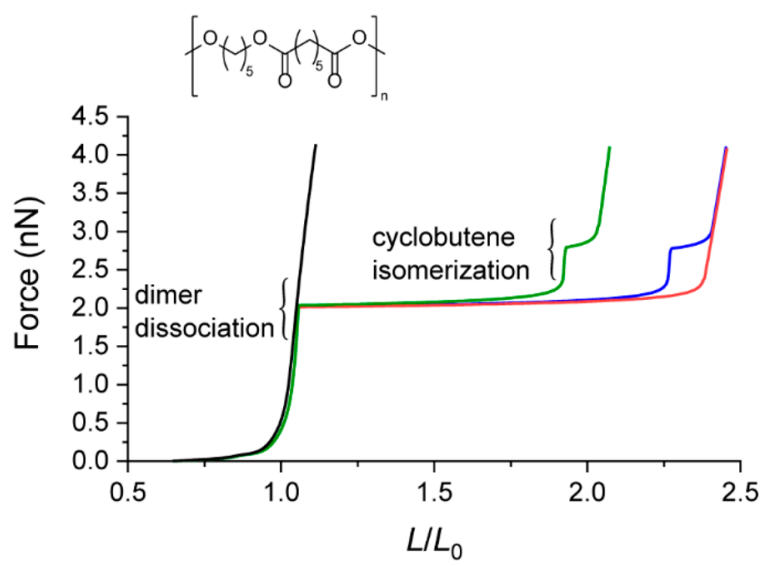

C

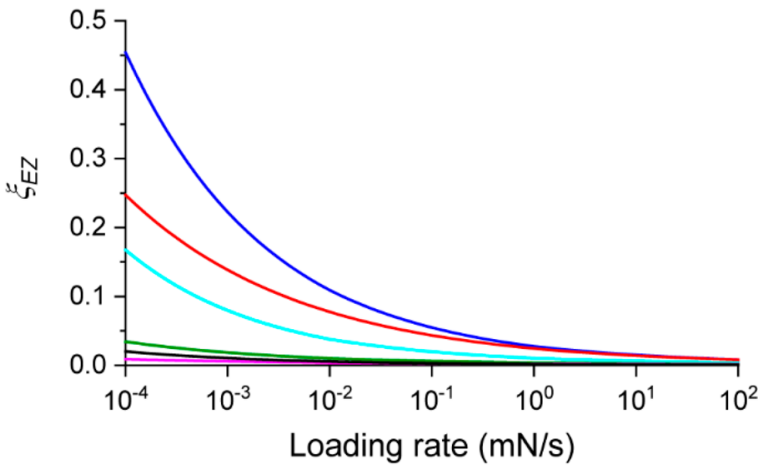

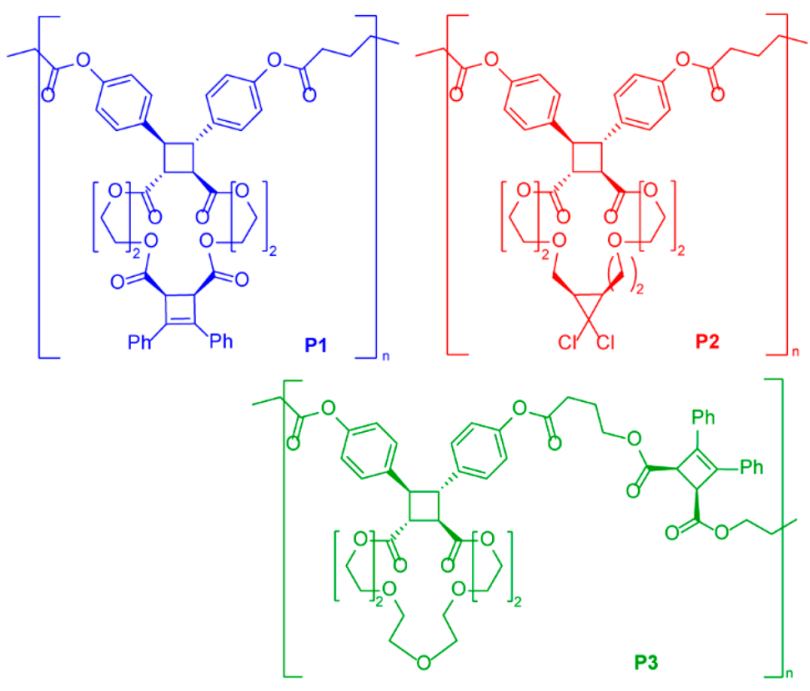

Figure 3. Summary of the computed mechanochemistry of tricyclic mechanophore $\mathbf{1}$ and its polymers. (A) Activation free energies as a function of the applied force for the key isomerization reactions at CAM-B3LYP/6-311+G(d) /CPCM = DMSO. For reactions marked with an asterisk, only the s conformers, which are the products of isomerization at force $>1 \mathrm{nN}$, are shown. At lower force Z-2 and E-2 yield h conformers (Figure 1). (B, C) Effect of the functional on the calculated selectivity of mechanochemical isomerization of $Z-2$, expressed as a fraction of $Z-2$ isomerizing to $E, Z$ diene, $\xi_{\mathrm{EZ}}$. (B) Under the assumption that the applied force does not change during the reaction, $\xi_{E Z}$ is a function of the half-life of $Z-2, \tau_{1 / 2}\left(10^{9} \mathrm{~s}\right.$ $\approx 32$ years). (C) Under the assumption that force increases linearly with reaction time, $\xi_{E Z}$ is a function of the constant loading rate. In this regime $\xi_{E Z}$ also depends on the reaction extent: the data shown are for $50 \%$ conversion. Both sets of curves are color-matched to the functionals listed in (B). (D) Calculated ensemble-average single-chain force/extension curves of hidden-length polymers P1-P3 and a mechanochemically inactive polyester. $L$ and $L_{0}$ are the stretched chain length and strain-free contour length of the intact polymer, respectively. The plateaus of curves lack the sawtooth pattern because the average chain is always in mechanical equilibrium with the applied force.

\section{RESULTS}

Quantum-Chemical Calculations of the Reaction Mechanisms and Kinetics of 2. Unlike the parent cyclobutene, whose thermal ${ }^{22-24}$ and mechanochemi$\mathrm{cal}^{20,25-27}$ dissociation has been studied extensively, little literature exists on the kinetics or mechanism of isomerization of diphenylcyclobutenes, such as 2 . Figure 2 illustrates the calculated mechanism of interconversion among the cyclo- butenes and the diene (muconic acid) isomers of 2 in the absence of applied force. The activation free energies calculated at the CAM-B3LYP/6-311+G(d) level of the DFT and DMSO-parametrized CPCM representation of the reaction solvent matched the experimental values to their measurement uncertainties (Table $\mathrm{S} 1$; mean-absolute deviation, MAD, of $0.7 \mathrm{kcal} / \mathrm{mol}$ ). MADs of the energies obtained with five additional functionals recommended for calculations 
Table 1. Effect of the Functional on the Calculated Contributions of the Competing Isomerization Mechanisms: the Calculated Minimum Force at Which the Open-Shell ("Symmetry Forbidden") Transition State Separating Z-2 from the (E,E)Diene Exists, $f_{\text {os }}{ }^{\mathrm{min}}$, the Maximum Force at Which the Closed-Shell ("Symmetry Allowed") Transition State Separating Z-2 from the $(E, Z)$-Diene exists, $f_{\mathrm{cs}}{ }^{\mathrm{max}}$, and the Force Window at Which the Two Transition States Are Kinetically Competitive, $\Delta f_{\mathrm{c}}^{a}$

\begin{tabular}{|c|c|c|c|c|c|c|}
\hline & \multicolumn{6}{|c|}{ functional } \\
\hline & APFD & CAM-B3LYP & M06-2X & wB97x-D & MWP1K & BMK \\
\hline$f_{\mathrm{os}}^{\min }(\mathrm{nN})$ & 2.0 & 1.7 & 2.0 & 1.5 & 1.2 & 1.9 \\
\hline$f_{\mathrm{cs}}^{\max }(\mathrm{nN})$ & 4.1 & 3.2 & 4.2 & 3.8 & 2.7 & 4.4 \\
\hline$\Delta f_{\mathrm{c}}(\mathrm{nN})$ & 0.7 & 0.9 & 0.9 & 0.9 & 1.3 & 1.1 \\
\hline
\end{tabular}

${ }^{a}$ All calculations of the open-shell structures were with the spin-unrestricted formalism.

of reaction barriers ${ }^{28,29}$ ranged from a low of $1.9 \mathrm{kcal} / \mathrm{mol}$ for M06-2X to a high of $3.6 \mathrm{kcal} / \mathrm{mol}$ for MPW1K (Table S1), primarily because these functionals overstabilize the cyclobutene isomers relative to the dienes. For all of the functionals, the $6-311+\mathrm{G}(\mathrm{d})$ basis set reduces the MAD relative to 6$31+\mathrm{G}(\mathrm{d})$, with the largest reduction (by $1.2 \mathrm{kcal} / \mathrm{mol}$ ) seen with CAM-B3LYP. Inclusion of a reaction solvent changed the activation free energies by $<0.5 \mathrm{kcal} / \mathrm{mol}$, with the barriers for direct isomerization of the $\mathrm{C}=\mathrm{C}$ bond being the most sensitive. Understanding the effect of model chemistry on the strain-free energies is important in predicting mechanochemical kinetics, as discussed below.

A mechanistically noteworthy result of these calculations is preferential $Z / E$ isomerization of the $h, E, Z$ and $h, Z, Z$ dienes through a pyran-type intermediates. A sequence of three steps - intramolecular cyclization, a flip of the resulting pyran ring, which switches the position of the $\mathrm{CO}_{2} \mathrm{Me}$ group from axial to equatorial, and its dissociation-lowers the $Z / E$ barrier by $>8 \mathrm{kcal} / \mathrm{mol}$ relative to single-step isomerization by rotation around the $\mathrm{C}=\mathrm{C}$ bond. Despite the biochemical and industrial importance of muconic acid derivatives, ${ }^{30}$ the possibility of such a facile multistep isomerization of a $\mathrm{C}-\mathrm{C}$ bond has not been previously recognized.

Tensile force acting at the $\mathrm{C}$ atoms of the methoxy groups modifies the reaction surface by

(1) eliminating the energy minima corresponding to the $h$ conformers of the dienes (second row, Figure 2)

(2) lowering the kinetic barriers for $E-2 \rightarrow$ s, $E, E$ diene, $Z$-2 $\rightarrow \mathrm{s}, E, Z$ diene and $\mathrm{s}, Z, Z \rightarrow \mathrm{s}, E, Z \rightarrow \mathrm{s}, E, E$ conversions (Figure 3) but increases the barriers separating $E-2$ from $\mathrm{s}, Z, Z$ diene (which requires an inward motion of the carboxylate groups during symmetry-allowed ring opening) and the pyran intermediates from both $s, E, Z$ and $\mathrm{s}, Z, Z$ dienes

(3) creating a new radicaloid singlet transition state connecting Z-2 and s,E,E diene and, at higher force, eliminating the barrier connecting $Z-2$ and s,E,Z diene.

The first two effects are expected on the basis of the simplest model of mechanochemical kinetics. ${ }^{31-33}$ In the absence of externally applied force, the $\mathrm{h}$ conformers reside in shallow energy minima, being separated from the $s$ analogues by free energy barriers $<10 \mathrm{kcal} / \mathrm{mol}$. Since the ${ }_{\mathrm{MeO}} \mathrm{C} \cdots \mathrm{C}_{\mathrm{MeO}}$ distance is shorter in the $\mathrm{h}$ conformers than in the $\mathrm{s}$ analogues (Table S2), tensile force reduces the depths of their energy well, ${ }^{32}$ eventually eliminating them at force of $0.1-0.8 \mathrm{nN}$, depending on the isomer. Similarly, $Z \rightarrow E$ isomerization of a $C=C$ double bond elongates the molecule and is thus accelerated by force. Conversely, the formation of the $Z, Z$ diene from $E-2$ and of the pyrene-type intermediates from $E, Z$ and $Z, Z$ dienes requires contraction of the ${ }_{\mathrm{MeO}} \mathrm{C} \cdots \mathrm{C}_{\mathrm{MeO}}$ distance in the corresponding transition states (Table S2) and hence is force-inhibited.

The nonmonotonic dependence of $\Delta G^{\ddagger}$ for isomerization of $Z-2$ to $E, Z$ diene and of $E-2$ to $E, E$ diene (red and green curves, Figure $3 \mathrm{~A}$ ) reflects the change with force in the minimumenergy conformers of the corresponding transition states. At forces of $<0.3$ and $<0.6 \mathrm{nN}$, respectively, these states are dominated by conformers with ${ }_{\mathrm{MeO}} \mathrm{C} \cdots \mathrm{C}_{\mathrm{MeO}}$ separations that are shorter than those in the corresponding cyclobutenes (Table S2). These short conformers are eliminated at 0.3 and $0.6 \mathrm{nN}$ for Z-2 and E-2, respectively, and at higher force, $\Delta G^{\ddagger}$ decreases nearly proportionally to force.

The unexpectedly weak sensitivity of the kinetics of isomerization between $E, Z$ and $E, E$ dicarboxyhexadienes to applied force, particularly at $>1 \mathrm{nN}$ (black curve, Figure 3A) results from the $E, Z$ isomer being softer (more deformable) than the $E, E$ analogue along the pulling axis. As a result, the elongation of the ${ }_{\mathrm{MeO}} \mathrm{C} \cdots \mathrm{C}_{\mathrm{MeO}}$ distance from the $E, Z$ isomer to the transition state for $E, Z \rightarrow E, E$ isomerization decreases from $1.4 \AA$ at $0 \mathrm{pN}$ to $0.1 \AA$ at $3 \mathrm{nN}$ and the activation barrier becomes proportionally less sensitive to force. A similar effect likely contributes to the distinct stereochemical outcomes of mechanochemical dissociation of dicarboxycyclobutanes. ${ }^{6,34}$

Highly nonlinear $\Delta G^{\ddagger}$ vs force correlations resulting from strong dependences of either the compositions of conformational ensembles or molecular compliances were noted in other reactions. $^{35-37}$ If such effects are common, as seems likely, they will be responsible for significant errors in the quantitative interpretation of single-molecule force measurements using Bell-Evans ansatzes. ${ }^{18,38,39}$

The effect of the force on the transition states separating Z-2 from the dienes (effect 3 above) again suggests a phenomenon more complex than is commonly discussed. ${ }^{20,26}$ First, the barrier for the symmetry-allowed, conratatory isomerization of $Z-2$ to $E, Z$ diene (red line) is nearly as sensitive to tensile force ( $1 \mathrm{kcal} / \mathrm{mol}$ reduction per $0.15-0.17 \mathrm{nN}$, depending on the functional) as that of the $E-2$ isomer (green line, $1 \mathrm{kcal} / \mathrm{mol}$ reduction per $0.13-0.15 \mathrm{nN}$ ). Consequently, an experimental observation of an accelerated dissociation of a $Z$ cyclobutene under tensile $\operatorname{load}^{26}$ may not mean that the dissociation proceeds by a reaction mechanism that is distinct from that in strain-free cyclobutenes or yields a different product. Second, the concerted conratatory and radicaloid (open-shell) transition states coexist over a $1.5-2 \mathrm{nN}$ range and are kinetically competitive over a $1 \mathrm{nN}$ range (Table 1 ) for any of the six functionals examined. In comparison, mechanistic discussions ${ }^{40}$ of mechanochemical isomerization of Z-benzocyclobutene have not invoked kinetic competition between isomerization mechanisms, suggesting instead that one path 
cleanly disappears above $0.8 \mathrm{nN}$. The stereoelectronic factors responsible for the potential difference between 2 and benzocyclobutene remain to be elucidated but may be related to the effect of planarity constraints on the allowed/forbidden gap in such isomerizations. ${ }^{41}$

The relative contributions of the two isomerization paths (symmetry allowed to $E, Z$ and symmetry forbidden to $E, E$ diene) to the overall rate of ring-opening of Z-2 are very sensitive to the functional, but not the basis set or the medium. The data are illustrated in Figure $3 \mathrm{~B}$ as a fraction of Z-2 dissociating to the $E, Z$ diene (symmetry-allowed conratatory mechanism), $\xi_{E Z}$, vs the half-life, $\tau_{1 / 2}$, of $Z$-2. For example, calculations with uMPW1K predict that $Z-2$ stretched to a constant force of $1.52 \mathrm{nN}$ isomerizes to an equimolar mixture of $E, Z$ and $E, E$ dienes $\left(\xi_{E Z}=0.5\right)$ with $\tau_{1 / 2} \approx 2.5$ years at 298 $\mathrm{K}$. Conversely, uM06-2X predicts that Z-2 isomerizes to the same mixture only if it is stretched with a much larger force $(2.65 \mathrm{nN})$, which reduces its $\tau_{1 / 2}$ value to just $30 \mathrm{~ms}$. In other words, the required decrease in the activation barrier needed to achieve the desired mechanochemical selectivity (in this example of 0.5 ) varies by $11 \mathrm{kcal} / \mathrm{mol}$ between these two functionals. In contrast, the activation free energies of isomerization of strain-free $Z$-2 calculated with the same pair of model chemistries vary by only $2.8 \mathrm{kcal} / \mathrm{mol}$ (Table S1). This suggests that accurate estimates of mechanochemical kinetics may require a functional that is different from one that reproduces the strain-free kinetics most accurately. If general, this observation underlines the importance of benchmarking computed mechanochemical kinetics against experiment. Because the volume of such experimental mechanochemical kinetic data is very limited, ${ }^{18}$ the findings in Figure $3 \mathrm{~B}$ suggest that special care is warranted in interpreting the computed mechanochemical reactivity.

The calculations of isomerization selectivity discussed above assumed, as is standard in contemporary polymer mechanochemistry, ${ }^{42}$ that every molecule of the reactant $(Z-2)$ in an ensemble is maintained at a constant force as the ensemble reacts. In many practical manifestations of mechanochemistry the ensemble-average force experienced by a reactant likely increases on a time scale comparable to $\tau_{1 / 2}{ }^{18}$ Data in Figure $3 \mathrm{C}$ account for this effect by recalculating the relative contribution of the symmetry-allowed path to isomerization of Z-2 under a constant loading rate (i.e., when the force increases linearly with reaction time).

At present we lack experimental data to compare with the trends in Figure 3B,C and thus to identify the functional that most accurately describes the mechanochemical kinetics of isomerization of $\mathbf{2}$. Sonication of the polymer of $\mathbf{1}, \mathbf{P 1}$, described in detail below, produced a mixture of $E, Z$ and $E, E$ dienes corresponding to $\xi_{E Z}=0.3$. While the microscopic conditions resulting in mechanochemistry in sonicated solutions are unknown, ${ }^{18}$ the transient nature of elongational flows responsible for it suggests that the reacting chains experience a loading rate of at least $1 \mathrm{mN} / \mathrm{s}$ and react on the sub-microsecond time scale. Only M06-2X predictions appear to be compatible with these limits. Dynamic single-molecule force spectroscopy (SMFS) allows reactions to be studied at loading rates of $10-100 \mathrm{nN} / \mathrm{s}$, but only if the half-lives can be reduced to $10-100 \mathrm{~ms} .{ }^{6,43}$ Unfortunately, the force required to reduce the $\tau_{1 / 2}$ value of $Z-2$ to this range $(2.7-3.0 \mathrm{nN}$, depending on the chain contour length, stretching rate, and model chemistry) exceeds the maximum force achievable in SMFS (see below).
Quantum-Chemical Calculations of Single-Chain Micromechanics. Previous studies demonstrated the value of understanding the micromechanics of isolated macromolecular chains in guiding the design of new materials that exploit the hidden-length mechanism. ${ }^{7}$ Using a described experimentally validated method, ${ }^{6,12,44}$ we calculated ensemble-average force/extension curves of three mechanochemically active polyesters and, for comparison, of a mechanochemically inert aliphatic polyester (Figure 3D), all of the same initial contour length. P1 is the polymer of mechanophore 1; in P2, the cyclobutene is replaced with a much more labile group, cisdichlorocyclopropane. P3 contains the same two mechanosensitive moieties as P1 (cinnamate dimer and cyclobutene) but connected serially, each contributing to the backbone of the intact chain. The length of the linkers within the cinnamate-dimer macrocycle in P2 and P3 was chosen so as to match each monomer's extensibility at $4 \mathrm{nN}$ to that of $\mathbf{1}$ at the same force.

The force/extension curves in Figure 3D were calculated for a stretching rate of $10 L_{0}$ per second, where $L_{0}$ is the strain-free contour length. Polymers P1-P3 manifest a common plateau at $\sim 2 \mathrm{nN}$ caused by mechanochemical dissociation of cinnamate dimers. An additional plateau at $\sim 2.75 \mathrm{nN}$ corresponding to the isomerization of cyclobutene to an approximately equimolar mixture of $E, Z$ and $E, E$ dienes is present only in P1 and P3. All force/extension curves terminate at $4.2 \mathrm{nN}$ due to chain fracture by homolysis of a backbone bond. From these curves we calculated the maximum chain extensibility (the ratio of the chain length at fracture to the strain-free contour length of the intact polymer) and chain fracture energy (the total area under the force/ extension curve) (Table 2). These parameters are single-chain equivalents of two key bulk mechanical properties of polymers, fractional elongation at break and fracture energy. ${ }^{45-47}$

Table 2. Summary of Calculated Single-Chain Micromechanical Properties of Polymers P1-P3 ${ }^{a}$

$\begin{array}{llll} & \text { P1 } & \text { P2 } & \text { P3 } \\ \text { chain extensibility }^{b} & 2.2 & 2.2 & 1.9 \\ \text { fracture energy }^{c} & 10.9 & 9.8 & 8.1\end{array}$

${ }^{a}$ The values are calculated relative to those of mechanochemically inert polyester (black, Figure 3D), all of the same initial strain-free contour length. ${ }^{b}$ Ratio of chain extensions of polymers P1-P3 and aliphatic polyester at chain fracture. ${ }^{c}$ Ratio of the area under the force/extension curve of polymers $\mathbf{P 1 - P 3}$ to that of aliphatic polyester up to chain fracture.

All hidden-length polymers sustain a fractional elongation 1.9-2.2 times that of a simple polyester of the same strain-free contour length without breaking and dissipate 8-11 times more mechanical (strain) energy at fracture, with P1 having the highest fracture energy. The higher fracture energy of P1 vs P2 illustrates that the mechanochemically active hidden length allows the chain fraction energy to be increased without changing chain extensibility or the ratio of the hidden length to the strain-free contour length in the intact polymer, which can affect the synthetic accessibility and processability of the polymer. ${ }^{48}$ The higher energy dissipating capacity of $\mathbf{P} \mathbf{1}$ results from the high force required for the hidden length to elongate irreversibly, because the moiety responsible for it (cyclobutene) is less mechanochemically labile than the primary sacrificial bond that releases hidden length. In contrast, the 
A<smiles>COC(=O)C1C(C(=O)OC)C(c2ccc(OC(C)=O)cc2I)C1c1ccc(OC(C)=O)cc1</smiles><smiles>COC(=O)/C=C/c1ccc(OC(C)=O)c(I)c1N</smiles><smiles>COC(=O)C1C(c2ccccc2)=C(c2ccccc2)C(C(=O)OC)C1C(=O)OC</smiles>
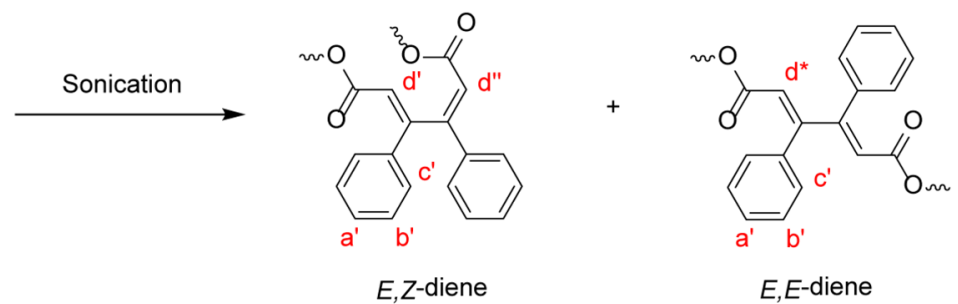

B

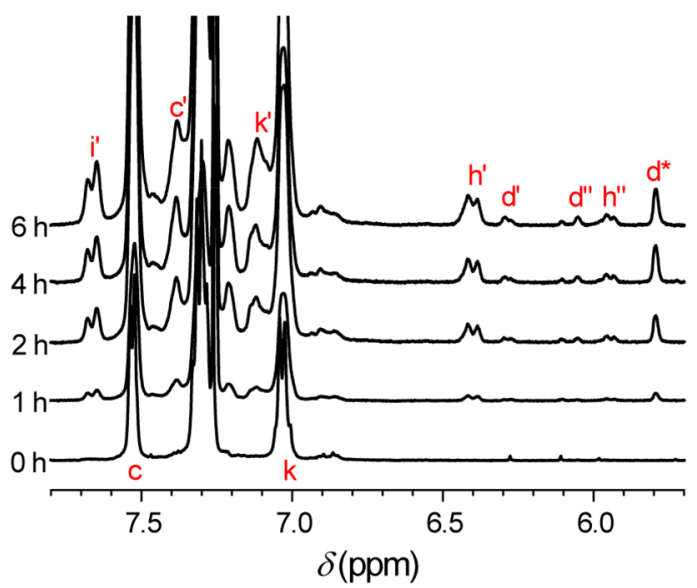

C

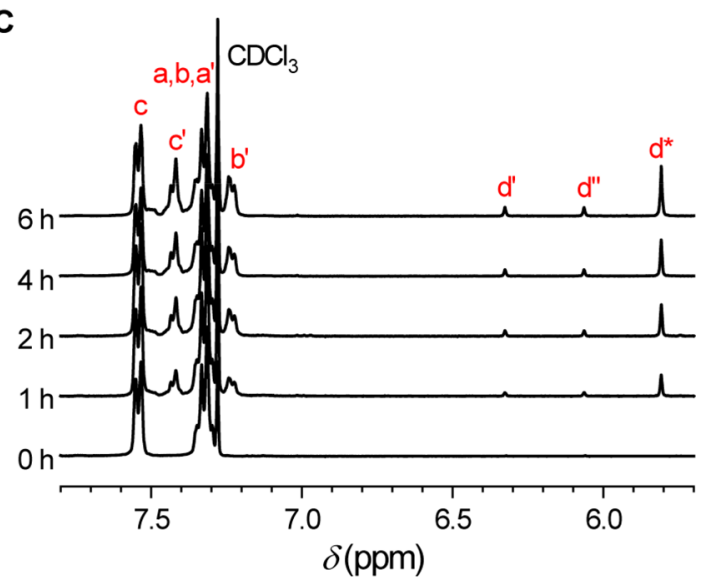

Figure 4. Spectroscopic evidence of mechanochemical reactions in ultrasonicated solutions of P1 and P4. (A) Molecular structures of the product moieties of mechanochemical reactions showing peak assignments. (B, C) Segments of the ${ }^{1} \mathrm{H}$ NMR spectra in $\mathrm{CDCl}_{3}$ of solutions of (B) P1 and (C) $\mathbf{P 4}$ at different sonication times. We assigned a multiplet at $5.95 \mathrm{ppm}$ (marked $\mathrm{h}^{\prime \prime}$ ) to cis-cinnamate produced by dissociation of a small fraction $(\sim 5 \%)$ of syn dimers in the sonicated polymer.

cyclopropane moiety responsible for the mechanochemical activity of the hidden length of $\mathbf{P 2}$ is more labile than the cinnamate dimer at any applied force, ${ }^{12}$ so that cyclopropane isomerizes to trans-2,3-dichloropropene simultaneously with dimer dissociation, and the resulting micromechanics, including fracture energy, is identical with that of a mechanochemically inert hidden length of the same extensibility.

The lower fracture energy of $\mathbf{P} \mathbf{3}$ relative to $\mathbf{P} \mathbf{1}$ reflects the lower extensibility of $\mathbf{P} \mathbf{3}$ necessitated by its serial arrangement of the two mechanosensitive moieties, which results in $40 \%$ fewer cinnamate dimers and cyclobutenes per unit length in comparison to P1. This illustrates how incorporating a second mechanophore into the hidden length instead of the backbone improves both chain extensibility and fracture energy in comparison to a simple copolymer of the two mechanophores with the same strain-free contour length.

Experimental Mechanochemistry of 1. We synthesized macrocycle 1 in three steps and $\sim 5 \%$ overall yield from commercial materials (Figures S1-S6). The overall yield is comparable to that of other cinnamate dimer macrocycles. ${ }^{6}$ The yield and the stereoselectivity of photodimerization of biscinnamates are sensitive to both the length of the linker separating the two dimerizing moieties and the concentration of $\mathrm{K}^{+}$, suggesting a templating effect. The linker connecting the two cinnamates in precursor 3 resulted in the selective generation of the anti dimer, which we favored to simplify the analysis of mechanochemical kinetics and reaction mixtures in the loaded materials. Copolymerization of 1 with excess glutaric acid under standard polyesterification conditions yielded polyester P1 $\left(M_{\mathrm{n}}=48 \mathrm{kDa}, \bigoplus_{\mathrm{M}}=1.7\right.$, Figures $\mathrm{S} 7$ and S8 and Table S3) for experimental studies of mechanochemistry of macrocycle $\mathbf{1}$. We applied the same polymerization method to a derivative of $Z-2,2^{\prime}$, to obtain the polyester of only diphenylcyclobutene, $\mathbf{P 4}\left(M_{\mathrm{n}}=33 \mathrm{kDa}, \bigoplus_{\mathrm{M}}=\right.$ 1.6, Figures S9 and S10A and Table S3) for comparison to P1.

Constant-velocity SMFS experiments followed a described protocol $^{6}$ on chains of P1 anchored by silicon-carbon bonds to a slide at one terminus and to an AFM tip at the other and stretched at a rate of $1 \mu \mathrm{m} / \mathrm{s}$ in DMF. The observed chain micromechanics was consistent with the results of quantumchemical calculations that cyclobutene is inert to a higher force than the dimer, which is key for using the mechanochemically active hidden length to increase the fracture energy at constant chain extensibility. The measured force/extension curves (Figure S11) reproduced closely the section of the calculated force/extension curves for chains of the same length at forces $<2.3 \mathrm{nN}$ (Figure 3D). Chain detachment prevented us from accessing higher forces required to isomerize cyclobutene on the time scale of an SMFS experiment. Consistent with this conclusion is the absence of detectable plateaus in force/ extension curves of the polyester of cyclobutene $Z-2^{\prime}$, P4 (see 
A

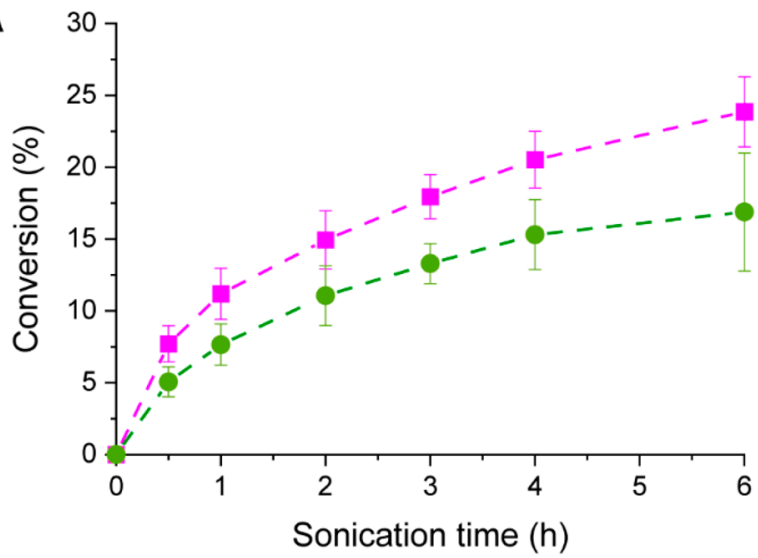

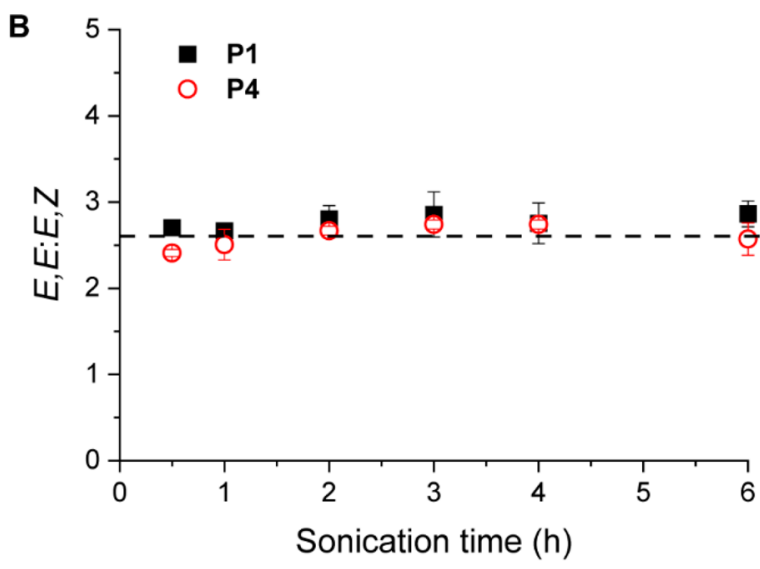

D

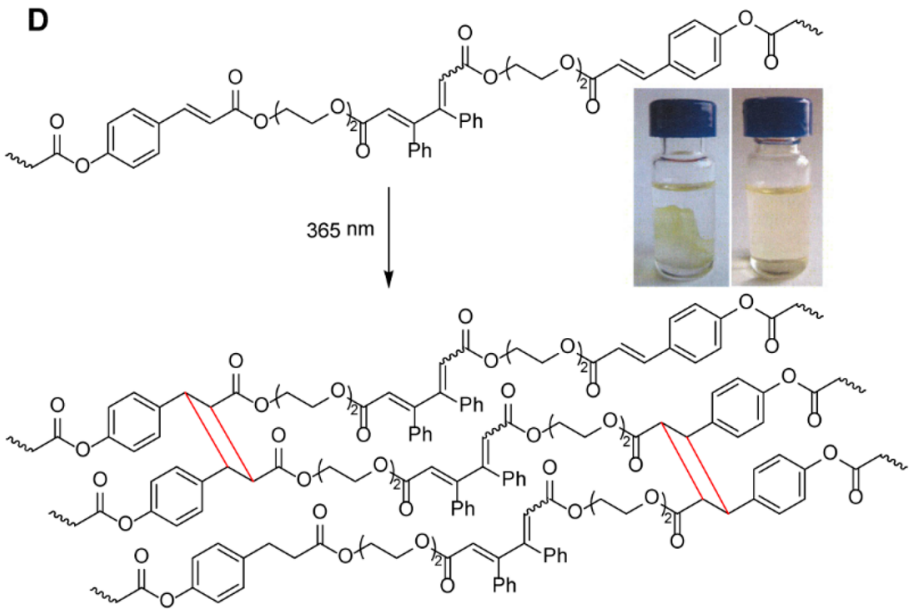

Figure 5. Summary of the experimental mechanochemistry of 1. (A) Fractions of dissociated cinnamate dimer (magenta) and isomerized cyclobutene (green) in P1 as a function of the sonication time. (B) Ratio of the two products of cyclobutene isomerization as a function of the sonication time for P1 and P4. Dashed lines are guides for the eyes. The error bars represent the standard deviation of three independent sonication measurements. Calculations of the fractions of conversion are described in the Supporting Information. (C) Increase in the UV absorbance at $280 \mathrm{~nm}$ due to cinnamate and diphenylhexadienedioic moieties as a function of sonication time of a THF solution of P1. (D) Irradiation of sonicated P1 at $365 \mathrm{~nm}$ creates an insoluble material (left vial in the inset) by photodimerization of cinnamates, whereas irradiation of either sonicated P4 (right vial in the inset) or intact P1, which lack cinnamate moieties, does not change the material solubility.

the Supporting Information), prior to chain detachment at 2.3-2.4 nN. As a result all force/extension curves of P1 resembled those of anti cinnamate dimers with mechanochemically inactive contour length. ${ }^{6}$ Across the 27 force/extension curves measured, the highest number of cinnamate dimers dissociated at an applied force of $1.75-1.80 \mathrm{nN}$ vs the calculated most probable force for the same set of chain lengths of 1.85-1.95 $\mathrm{nN}$ (Figure S12A). Dissociation of an average dimer resulted in the chain elongation by $2.9 \pm 0.7 \mathrm{~nm}$ in an experiment vs $2.86 \mathrm{~nm}$ (at $1.9 \mathrm{nN}$ ) (Figure S12B) from quantum-chemical calculations.

In contrast to SMFS experiments, sonication of dilute THF solutions of P1 $\left(M_{\mathrm{n}}=48 \mathrm{kDa}, \bigoplus_{\mathrm{M}}=1.7\right)$ under the standard conditions ${ }^{14,19,42}$ effected both mechanochemical reactions: dissociation of the dimers and isomerization of the cyclobutene. We monitored the composition of the sonicated solutions by periodically withdrawing aliquots, evaporating the solvent, redissolving the solids in $\mathrm{CDCl}_{3}$, and analyzing them by ${ }^{1} \mathrm{H}$ NMR spectroscopy. The spectra indicated a gradual growth in the relative intensity of the resonances of the cinnamic and diphenylhexadienedioic moieties and a corresponding decrease in the signals of the dimer and cyclobutene (Figure 4). We assigned resonances at $\delta 7.65$ and $6.42 \mathrm{ppm}$ (marked $\mathrm{i}^{\prime}$ and $\mathrm{h}^{\prime}$, respectively in Figure 4) to $E$ cinnamate on the basis of the literature data. ${ }^{6}$ The ${ }^{1} \mathrm{H}$ NMR spectra of sonicated solutions contained peaks of both $E, Z(\delta 6.33$ and $6.07 \mathrm{ppm}$, marked $\mathrm{d}^{\prime}$ and $\mathrm{d}^{\prime \prime}$ in Figure $\left.4 \mathrm{~B}, \mathrm{C}\right)$ and $E, E$ isomers ( $\delta$ 5.81 ppm, marked $\mathrm{d}^{*}$ in Figure 4B,C) of the diphenylhexadienedioic moiety. We confirmed this assignment by sonicating a solution of P4 $\left(M_{\mathrm{n}}=33 \mathrm{kDa}, \bigoplus_{\mathrm{M}}=1.6\right)$, which contains only Z-diphenylcyclobutene and thus yields simpler NMR spectra in comparison to P1. The aromatic protons of diphenylcyclobutene or its diene isomers are obscured by those of cinnamate in the spectra of $\mathbf{P 1}$ but are distinguishable in the spectra of $\mathbf{P 4}$ and its sonicated solutions.

The well-separated ${ }^{1} \mathrm{H}$ NMR resonances of the products of mechanochemical reactions (cinnamates, $E, Z$ and $E, E$ dienes) allowed us to quantify the reaction selectivities, which were all independent of the reaction extent (Figure 5). Thus, at all sonication times, the ratio of dissociated cinnamate dimer to isomerized cyclobutene was $1.4 \pm 0.1$, corresponding to one cyclobutene moiety isomerizing per 1.4 dissociated dimers (Figure S13). Although the origin of this ratio remains to be established, two plausible explanations are that (a) a subset of polymer chains is only stretched enough to dissociate the more labile dimer but not to isomerize the cyclobutene before the transient flow field dissipates or (b) it reflects a kinetic competition between cyclobutene isomerization and non- 
selective backbone fracture in chains containing dissociated dimers. Such fractured chains are likely to be too short to again become sufficiently stretched for the cyclobutene to isomerize. Qualitatively, the chain fracture is expected to become increasingly competitive as the loading rates increase. ${ }^{18}$ Quantitative assessment of these possibilities must await reliable estimates of the magnitudes of loading rates experienced by reacting chains in elongational flows of collapsing cavitation bubbles, which are currently lacking.

The ratio of $E, E$ diene to $E, Z$ diene was $0.73 \pm 0.01$ in the sonication of P1 and P4, regardless of the sonication time. The high calculated barrier of mechanochemical $E, Z \rightarrow E, E$ isomerization of the dienes at any force up to chain fracture (black line, Figure 3A) makes it highly unlikely that the E,E product resulted from sequential isomerization of cyclobutene to the $E, Z$ diene, followed by isomerization of the $E, Z$ diene to the $E, E$ analogue. The identical ratio in two different polymers argues against the two products resulting from two sets of different reaction conditions. ${ }^{18}$ Consequently, the measured ratio suggests that the symmetry-allowed mechanism accounts for $27 \%$ of isomerization, with the rest following the symmetryforbidden path.

The mechanochemical nature of these reactions is evidenced by the absence of any changes (Figure S14) in the composition of a sonicated solution of a short analogue of P4 $\left(M_{n}=6.8\right.$ $\mathrm{kDa}, \bigoplus_{\mathrm{M}}=1.4$, Figure S10B and Table S3), because only mechanochemical, not purely thermal, kinetics depends strongly on the polymer length.

Both dissociation of the cinnamate dimer and isomerization of diphenylcyclobutene are mechanochromic: i.e., they produce species which absorb at higher wavelengths in comparison to the reactants (Figure 5C). Because cinnamates photodimerize, mechanochemically remodeled materials containing cinnamates are optically healable. Importantly, the presence of diphenylcyclobutene or its diene does not interfere with the formation of new cyclobutanes under UV irradiation despite overlapping absorption of the two chromophores. We sonicated a sample of $\mathbf{P 1}$ until its average molar mass halved by nonselective backbone fragmentation that always competes with productive chemistry in sonicated polymer solutions (Figure S15 and Table S4). ${ }^{18}$ After removing the solvent, we irradiated the resulting solid for $3 \mathrm{~h}$ at $365 \mathrm{~nm}$ and an optical flux of $1.68 \mathrm{~W} / \mathrm{cm}^{2}$. The irradiated solid was insoluble in THF and all other organic solvents tested, which we ascribe to crosslinking of fragmented chains by photochemical $[2+2]$ cycloaddition of mechanically generated cinnamates. This behavior is identical with that of a polymer of cinnamates alone. In contrast, repeating the same procedure for polymer P4, whose sonication produces diphenylhexadienedioic but not cinnamic moieties, or irradiating nonsonicated P1 did not change the solubility of the irradiated solid (Figure 5D inset).

\section{DISCUSSION}

Although polymers containing a single (or single type of) mechanophore are the mainstay of contemporary polymer mechanochemistry, the advantages of combining multiple mechanochemical reactions in a single macrochain are becoming increasingly clear. ${ }^{1,16}$ For example, dual-mechanophore polymers have enabled fundamental quantitative studies of relative mechanochemical reactivities. ${ }^{49}$ Our work demonstrates that the spatial arrangement of the distinct mechanophores is at least as important as their nature, as illustrated by the much greater extensibility and fracture energy of polymer
P1 in comparison to the compositionally equivalent P3 (Figure 3D), in which the same two mechanophores are connected in parallel (P1) or in series (P3). Only the parallel arrangement, producing a mechanochemically active hidden length, permits independent changes in the chain extensibility and chain fracture energy by controlling the fraction of the total chain elongation that occurs at higher force and the difference in mechanochemical kinetics of the two mechanosensitive moieties.

Whether the more stable of the two mechanophores holds the hidden length (sacrificial moiety) or is incorporated into the hidden-length loop directly affects chain micromechanics, as illustrated by force/extension curves of polymers P1 and P2 (Figure 3D). The two polymers have the same sacrificial moiety (cyclobutane) and identical extensibilities, but the mechanical stabilities of their hidden lengths differ by $\sim 2 \mathrm{nN}$. If the goal is to increase the fracture energy, the more inert mechanophore should be incorporated in the hidden length (P1), whereas using the more inert mechanophore as the sacrificial moiety (P2) increases the maximum force to which the hidden length remains mechanochemically inert (mechanochemical gating ${ }^{12,14}$ ).

The fact that both scenarios have now been demonstrated experimentally using the same sacrificial moiety (cyclobutane) reflects the favorable mechanochemical stability and synthetic accessibility of cyclobutane derivatives. The current molecular architecture exemplified by mechanophore 1 improves the original bicyclooctane design ${ }^{12}$ by enabling diverse mechanophores to be incorporated into the hidden length with minimal incremental synthetic effort. The high mechanochemical stability of the cyclobutane core is valuable in increasing chain fracture energy, but it creates a challenge of identifying reactive sites that are even inert to even higher force, which were unknown prior to our work.

The new mechanophore Z-2,3-diphenyl-cyclobutene-1,4dicarboxylate is stable for $>1 \mathrm{~s}$ at $>2.5 \mathrm{nN}$, which is responsible for the high fracture energy of $\mathbf{P 1}$ and the unprecedented range of forces over which P1 is capable of dissipating mechanical energy without fracture. The mechanochemical isomerization of this cyclobutene in sonicated solutions yields an approximately equimolar mixture of two dienes. The result is consistent with the quantum-chemical calculations that two isomerization mechanisms, formally "symmetry allowed" and "symmetry forbidden" yielding the two observed dienes, are kinetically competitive over a $>1 \mathrm{nN}$ range of applied forces. Although the possibility of chain fracture by competing homolytic and heterolytic scissions of a backbone bond, usually with solvent contribution, has long been recognized, Z2 appears to be the only nonscissile mechanophore demonstrated to react by two well-defined competing mechanisms under any loading conditions. In sonicated solutions, dicarboxycyclobutanes dissociate to a mixture of isomeric olefins, ${ }^{34}$ but a mechanistic interpretation of the results remains challenging.

Force-dependent product distributions of 1 suggest that 1 may form a basis for a general quantitative method of estimating single-chain forces experiences by polymers in solutions, melts, and solids under a mechanical load, which is one of the more intractable problems in modern polymer mechanochemistry. ${ }^{50}$ A mechanophore that generates distinct species depending on the applied force, which our bifunctional macrocycle does, allows this force to be estimated from the composition of the product mixture. Depending on the force, 
in $\mathbf{1}$ the mechanochemical product mixture would contain, in addition to the cinnamate product of dimer dissociation, intact cyclobutene, E,Z diene, and/or E,E diene. Because of the negligibly slow interconversion between $E, Z$ and $E, E$ products (Figure $3 \mathrm{~A}$ ), the fractions of intact cinnamate dimers, cyclobutenes, and $E, Z$ and $E, E$ dienes in a mechanically loaded material in theory reflect the forces experienced by an average chain in the corresponding volume of the material. At present, usefully accurate estimates of the force (or loading rate) responsible for the observed reaction stoichiometry are precluded by the uncertainty of the specific force range over which the two isomerization mechanisms of cyclobutene compete. Our ongoing effort to achieve cyclobutene isomerization in SMFS by developing new anchoring chemistry and to prepare a series of analogues of $\mathbf{1}$ with varying hidden lengths is designed to enable such estimates.

\section{CONCLUSIONS}

In conclusion, we have reported computational and experimental evidence of the potential benefits of making the polymer hidden length mechanochemically active, including a means of independently controlling chain extensibility and chain fracture energy and of integrating additional productive mechanochemical responses into the polymer. Our new mechanophore incorporates Z-2,3-diphenylcyclobutene-1,4dicarboxylate, with hitherto unknown mechanochemistry, into macrocyclic cinnamate dimers. A polymer of this tricyclic mechanophore more than doubles its contour length without fracture when it is stretched and retains stress-dissipating capacity up to $3 \mathrm{nN}$ of applied force, which results in a chain fracture energy 11 times that of a simple polyester of the same initial contour length. In sonicated solutions mechanochemical isomerization of the cyclobutene yields two dienes, confirming our quantum-chemical calculations that two isomerization mechanisms, formally "symmetry allowed" and "symmetry forbidden", are kinetically competitive over a $>1 \mathrm{nN}$ range of applied forces. These calculations also indicate that DFT functionals with comparable capacities to reproduce strain-free kinetics yield highly divergent results for kinetics under force. This finding emphasizes the importance of validating computational predictions of mechanochemistry against experiment.

\section{ASSOCIATED CONTENT}

\section{SI Supporting Information}

The Supporting Information is available free of charge at https://pubs.acs.org/doi/10.1021/jacs.0c09220.

Quantum-chemical calculations, detailed experimental procedures, characterization, and additional SMFS (PDF)

\section{AUTHOR INFORMATION}

\section{Corresponding Authors}

Wengui Weng - Department of Chemistry, College of Chemistry and Engineering, Xiamen University, Xiamen, Fujian 361005, People's Republic of China; (1) orcid.org/0000-0003-31443181; Email: wgweng@xmu.edu.cn

Wenke Zhang - State Key Laboratory of Supramolecular Structure and Materials, College of Chemistry, Jilin University, Changchun, Jilin 130012, People's Republic of China; 이이.org/0000-0002-4569-6035; Email: zhangwk@ jlu.edu.cn
Roman Boulatov - Department of Chemistry, University of Liverpool, Liverpool L69 7ZD, U.K.; 이이이.org/0000-00027601-4279; Email: r.boulatov@liverpool.ac.uk

\section{Authors}

Yancong Tian - Department of Chemistry, University of Liverpool, Liverpool L69 7ZD, U.K.

Xiaodong Cao - Department of Chemistry, College of Chemistry and Engineering, Xiamen University, Xiamen, Fujian 361005, People's Republic of China

Xun Li - State Key Laboratory of Supramolecular Structure and Materials, College of Chemistry, Jilin University, Changchun, Jilin 130012, People's Republic of China

Huan Zhang - Department of Chemistry, College of Chemistry and Engineering, Xiamen University, Xiamen, Fujian 361005, People's Republic of China

Cai-Li Sun - Department of Chemistry, University of Liverpool,

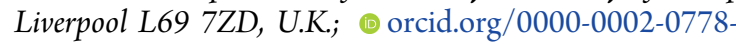
2004

Yuanze Xu - Department of Chemistry, College of Chemistry and Engineering, Xiamen University, Xiamen, Fujian 361005, People's Republic of China

Complete contact information is available at:

https://pubs.acs.org/10.1021/jacs.0c09220

\section{Author Contributions}

${ }$ Y.T., X.C., and X.L. contributed equally.

\section{Notes}

The authors declare no competing financial interest.

\section{ACKNOWLEDGMENTS}

X.C., H.Z., Y.X., and W.W. are funded by the National Natural Science Foundation of China (No. 21774106, No. 21574108). X.L. and W.Z. are funded by the NNSFC (No. 21525148) and the National Basic Research Program (No. 2013CB834503). Y.T., C.-L.S., and R.B. acknowledge funding from the UK Engineering and Physical Sciences Research Council (EP/ L000075/1). Calculations described here used the Extreme Science and Engineering Discovery Environment (XSEDE), which is supported by the National Science Foundation grant number ACI-1548562, with computational resources provided by the SDSC under allocation TG-CHE140039. Acknowledgment is made to the donors of the American Chemical Society Petroleum Research Fund for partial support of this research under grant 58885-ND7.

\section{REFERENCES}

(1) Brown, C. L.; Craig, S. L. Molecular engineering of mechanophore activity for stress-responsive polymeric materials. Chem. Sci. 2015, 6 (4), 2158-2165.

(2) Lieou, C. K. C.; Elbanna, A. E.; Carlson, J. M. Sacrificial bonds and hidden length in biomaterials: A kinetic constitutive description of strength and toughness in bone. Physical Review E - Statistical, Nonlinear, and Soft Matter Physics 2013, 88 (1), No. 012703.

(3) Kean, Z. S.; Craig, S. L. Mechanochemical remodeling of synthetic polymers. Polymer 2012, 53 (5), 1035-1048.

(4) Fantner, G. E.; Hassenkam, T.; Kindt, J. H.; Weaver, J. C.; Birkedal, H.; Pechenik, L.; Cutroni, J. A.; Cidade, G. A. G.; Stucky, G. D.; Morse, D. E.; Hansma, P. K. Sacrificial bonds and hidden length dissipate energy as mineralized fibrils separate during bone fracture. Nat. Mater. 2005, 4 (8), 612-616.

(5) Bao, Y.; Luo, Z.; Cui, S. Environment-dependent single-chain mechanics of synthetic polymers and biomacromolecules by atomic force microscopy-based single-molecule force spectroscopy and the 
implications for advanced polymer materials. Chem. Soc. Rev. 2020, 49 (9), 2799-2827.

(6) Zhang, H.; Li, X.; Lin, Y.; Gao, F.; Tang, Z.; Su, P.; Zhang, W.; $\mathrm{Xu}, \mathrm{Y}$.; Weng, W.; Boulatov, R. Multi-modal mechanophores based on cinnamate dimers. Nat. Commun. 2017, 8 (1), 1147.

(7) Chung, J.; Kushner, A. M.; Weisman, A. C.; Guan, Z. Direct correlation of single-molecule properties with bulk mechanical performance for the biomimetic design of polymers. Nat. Mater. 2014, 13 (11), 1055-1062.

(8) Wu, D.; Lenhardt, J. M.; Black, A. L.; Akhremitchev, B. B.; Craig, S. L. Molecular Stress Relief through a Force-Induced Irreversible Extension in Polymer Contour Length. J. Am. Chem. Soc. 2010, 132 (45), 15936-15938.

(9) Huang, W.; Restrepo, D.; Jung, J.-Y.; Su, F. Y.; Liu, Z.; Ritchie, R. O.; McKittrick, J.; Zavattieri, P.; Kisailus, D. Multiscale toughening mechanisms in biological materials and bioinspired designs. Adv. Mater. (Weinheim, Ger.) 2019, 31 (43), 1901561.

(10) Mazzotta, M. G.; Putnam, A. A.; North, M. A.; Wilker, J. J. Weak Bonds in a Biomimetic Adhesive Enhance Toughness and Performance. J. Am. Chem. Soc. 2020, 142 (10), 4762-4768.

(11) Meyers, M. A.; McKittrick, J.; Chen, P. Y. Structural biological materials: Critical mechanics-materials connections. Science 2013, 339 (6121), 773-779.

(12) Wang, J.; Kouznetsova, T. B.; Boulatov, R.; Craig, S. L. Mechanical gating of a mechanochemical reaction cascade. Nat. Commun. 2016, 7, 13433.

(13) Wang, F.; Diesendruck, C. E. Effect of disulphide loop length on mechanochemical structural stability of macromolecules. Chem. Commun. 2020, 56 (14), 2143-2146.

(14) Lin, Y.; Kouznetsova, T. B.; Craig, S. L. Mechanically Gated Degradable Polymers. J. Am. Chem. Soc. 2020, 142 (5), 2105-2109.

(15) Ducrot, E.; Chen, Y.; Bulters, M.; Sijbesma, R. P.; Creton, C. Toughening Elastomers with Sacrificial Bonds and Watching Them Break. Science (Washington, DC, U. S.) 2014, 344 (6180), 186-189.

(16) Chen, Z.; Mercer, J. A. M.; Zhu, X.; Romaniuk, J. A. H.; Pfattner, R.; Cegelski, L.; Martinez, T. J.; Burns, N. Z.; Xia, Y. Mechanochemical unzipping of insulating polyladderene to semiconducting polyacetylene. Science 2017, 357 (6350), 475.

(17) Schütze, D.; Holz, K.; Müller, J.; Beyer, M. K.; Lüning, U.; Hartke, B. Pinpointing Mechanochemical Bond Rupture by Embedding the Mechanophore into a Macrocycle. Angew. Chem., Int. Ed. 2015, 54 (8), 2556-2559.

(18) Akbulatov, S.; Boulatov, R. Experimental Polymer Mechanochemistry and its Interpretational Frameworks. ChemPhysChem 2017, 18 (11), 1422-1450.

(19) Izak-Nau, E.; Campagna, D.; Baumann, C.; Göstl, R. Polymer mechanochemistry-enabled pericyclic reactions. Polym. Chem. 2020, 11 (13), 2274-2299.

(20) Kochhar, G. S.; Heverly-Coulson, G. S.; Mosey, N. J. Theoretical approaches for understanding the interplay between stress and chemical reactivity. Top. Curr. Chem. 2015, 369, 37-96.

(21) Wang, J.; Kouznetsova, T. B.; Craig, S. L. Reactivity and Mechanism of a Mechanically Activated anti-Woodward-HoffmannDePuy Reaction. J. Am. Chem. Soc. 2015, 137 (36), 11554-11557.

(22) Kirmse, W.; Rondan, N. G.; Houk, K. N. Stereoselective substituent effects on conrotatory electrocyclic reactions of cyclobutenes. J. Am. Chem. Soc. 1984, 106 (25), 7989-7991.

(23) Dolbier, W. R., Jr.; Koroniak, H.; Houk, K. N.; Sheu, C. Electronic Control of Stereoselectivities of Electrocyclic Reactions of Cyclobutenes: A Triumph of Theory in the Prediction of Organic Reactions. Acc. Chem. Res. 1996, 29 (10), 471-477.

(24) Um, J. M.; Xu, H.; Houk, K. N.; Tang, W. Thermodynamic Control of the Electrocyclic Ring Opening of Cyclobutenes: $\mathrm{C}=\mathrm{X}$ Substituents at C-3 Mask the Kinetic Torquoselectivity. J. Am. Chem. Soc. 2009, 131 (19), 6664-6665.

(25) Yang, Q.-Z.; Huang, Z.; Kucharski, T. J.; Khvostichenko, D.; Chen, J.; Boulatov, R. A molecular force probe. Nat. Nanotechnol. 2009, 4 (5), 302-306.
(26) Wang, J.; Kouznetsova, T. B.; Niu, Z.; Ong, M. T.; Klukovich, H. M.; Rheingold, A. L.; Martinez, T. J.; Craig, S. L. Inducing and quantifying forbidden reactivity with single-molecule polymer mechanochemistry. Nat. Chem. 2015, 7, 323-327.

(27) Tian, Y.; Boulatov, R. Quantum-Chemical Validation of the Local Assumption of Chemomechanics for a Unimolecular Reaction. ChemPhysChem 2012, 13 (9), 2277-2281.

(28) Peverati, R.; Truhlar, D. G. Quest for a universal density functional: the accuracy of density functionals across a broad spectrum of databases in chemistry and physics. Philos. Trans. $R$. Soc., A 2014, 372 (2011), 20120476.

(29) Mardirossian, N.; Head-Gordon, M. Thirty years of density functional theory in computational chemistry: an overview and extensive assessment of 200 density functionals. Mol. Phys. 2017, 115 (19), 2315-2372.

(30) Khalil, I.; Quintens, G.; Junkers, T.; Dusselier, M. Muconic acid isomers as platform chemicals and monomers in the biobased economy. Green Chem. 2020, 22 (5), 1517-1541.

(31) Tian, Y.; Kucharski, T. J.; Yang, Q.-Z.; Boulatov, R. Model studies of force-dependent kinetics of multi-barrier reactions. Nat. Commun. 2013, 4 (1), 2538.

(32) Boulatov, R. Reaction dynamics in the formidable gap. Pure Appl. Chem. 2010, 83, 25-41.

(33) Kucharski, T. J.; Boulatov, R. The physical chemistry of mechanoresponsive polymers. J. Mater. Chem. 2011, 21, 8237-8255.

(34) Kean, Z. S.; Niu, Z.; Hewage, G. B.; Rheingold, A. L.; Craig, S. L. Stress-Responsive Polymers Containing Cyclobutane Core Mechanophores: Reactivity and Mechanistic Insights. J. Am. Chem. Soc. 2013, 135 (36), 13598-13604.

(35) Hermes, M.; Boulatov, R. The Entropic and Enthalpic Contributions to Force-Dependent Dissociation Kinetics of the Pyrophosphate Bond. J. Am. Chem. Soc. 2011, 133, 20044-20047.

(36) Akbulatov, S.; Tian, Y.; Kapustin, E.; Boulatov, R. Model Studies of the Kinetics of Ester Hydrolysis under Stretching Force. Angew. Chem., Int. Ed. 2013, 52, 6992-6995.

(37) Akbulatov, S.; Tian, Y.; Huang, Z.; Kucharski, T. J.; Yang, Q.Z.; Boulatov, R. Experimentally realized mechanochemistry distinct from force-accelerated scission of loaded bonds. Science 2017, 357 (6348), 299-303.

(38) Kouznetsova, T. B.; Wang, J.; Craig, S. L. Combined ConstantForce and Constant-Velocity Single-Molecule Force Spectroscopy of the Conrotatory Ring Opening Reaction of Benzocyclobutene. ChemPhysChem 2017, 18 (11), 1486-1489.

(39) Tian, Y.; Boulatov, R. Comparison of the predictive performance of the Bell-Evans, Taylor-expansion and statisticalmechanics models of mechanochemistry. Chem. Commun. 2013, 49, 4187-4189.

(40) Ribas-Arino, J.; Shiga, M.; Marx, D. Unravelling the Mechanism of Force-Induced Ring-Opening of Benzocyclobutenes. Chem. - Eur. J. 2009, 15 (48), 13331-13335.

(41) Lee, P. S.; Sakai, S.; Hoerstermann, P.; Roth, W. R.; Kallel, E. A.; Houk, K. N. Altering the Allowed/Forbidden Gap in Cyclobutene Electrocyclic Reactions: Experimental and Theoretical Evaluations of the Effect of Planarity Constraints. J. Am. Chem. Soc. 2003, 125 (19), $5839-5848$

(42) Anderson, L.; Boulatov, R. Polymer Mechanochemistry: A New Frontier for Physical Organic Chemistry. Adv. Phys. Org. Chem. 2018, $52,87-143$.

(43) Barbee, M. H.; Kouznetsova, T.; Barrett, S. L.; Gossweiler, G. R.; Lin, Y.; Rastogi, S. K.; Brittain, W. J.; Craig, S. L. Substituent Effects and Mechanism in a Mechanochemical Reaction. J. Am. Chem. Soc. 2018, 140 (40), 12746-12750.

(44) Akbulatov, S.; Tian, Y.; Boulatov, R. Force-Reactivity Property of a Single Monomer Is Sufficient To Predict the Micromechanical Behavior of Its Polymer. J. Am. Chem. Soc. 2012, 134, 7620-7623.

(45) Creton, C. 50th Anniversary Perspective: Networks and Gels: Soft but Dynamic and Tough. Macromolecules (Washington, DC, U. S.) 2017, 50 (21), 8297-8316. 
(46) Zhao, X. Multi-scale multi-mechanism design of tough hydrogels: building dissipation into stretchy networks. Soft Matter 2014, 10 (5), 672-687.

(47) Zhang, H.; Zeng, D.; Pan, Y.; Chen, Y.; Ruan, Y.; Xu, Y.; Boulatov, R.; Creton, C.; Weng, W. Mechanochromism and optical remodeling of multi-network elastomers containing anthracene dimers. Chem. Sci. 2019, 10 (36), 8367-8373.

(48) Li, M.; Zhang, H.; Gao, F.; Tang, Z.; Zeng, D.; Pan, Y.; Su, P.; Ruan, Y.; Xu, Y.; Weng, W. A cyclic cinnamate dimer mechanophore for multimodal stress responsive and mechanically adaptable polymeric materials. Polym. Chem. 2019, 10 (7), 905-910.

(49) Lee, B.; Niu, Z.; Wang, J.; Slebodnick, C.; Craig, S. L. Relative Mechanical Strengths of Weak Bonds in Sonochemical Polymer Mechanochemistry. J. Am. Chem. Soc. 2015, 137 (33), 10826-10832.

(50) Boulatov, R. The challenges and opportunities of polymer mechanochemistry. ChemPhysChem 2017, 18, 1419-1421. 